\title{
Avaliação de um serviço de atenção integral à saúde do adolescente
}

\author{
Evaluation of a comprehensive \\ adolescent health care service
}

Vera Lúcia Almei da Formigli 1

Maria Conceição Olivei ra Costa 2

Lauro Antonio Porto 1,3

\footnotetext{
1 Departamento de Medicina Preventiva, Universidade Federal da Bahia. Rua Reitor Miguel Calmon s/no, Salvador, BA 40110-100, Brasil. formigli@ufba.br lauroporto@uol.com.br 2 Departamento de Saúde, Universidade Estadual de Feira de Santana. BR 116, Km 3, Campus Universitário, Feira de Santana, BA 44031-460, Brasil. nepa@zipmail.com.br 3 Secretaria da Saúde do Estado da Bahia. Centro Administrativo da Bahia, 4a Avenida, Plataforma 6, Lado B, Salvador, BA 41750-300, Brasil.
}

Abstract Theauthors evaluate the Adolescent Heal th Clinic in the Emaús community in Belém, Pará, Brazil with regard to coverage, adequacy, accessibility, and utilization for the period 1994 to 1996. Coverage was calculated on the basis of clinical records and information collected from the target population. Adequacy was analyzed by comparing the service performance with goals established by PAHO/WHO for this kind of institution. Accessi bility and utilization patterns were evaluated from information obtained by a population-based survey among adolescents living in the area. Results showed: a good degree of program adequacy vis-à-vis the final purpose, although some adjustments are needed, especially in human resources; lack of barriers to user access; reasonable coverage, despite the low proportion of consultations by adolescents; and utilization pattern compatible with the service provision profile, mainly directed towards curative and individual care. Recommendations are made to revise the hegemonic health care model to emphasi ze preventive, collective, and educational activities.

Key words Adolescent Health; Health Service; Evaluation; Health Service Coverage

Resumo Foram avaliados a cobertura, a adequação, a acessibilidade e o padrão de utilização do Serviço de Saúde dos Adolescentes da comunidade de Emaús, Belém, Pará, no período de 1994 a 1996. A cobertura foi estimada com base nos registros da unidade de saúde e de informações col hidas junto à população-alvo; a adequação foi analisada por comparação com as condições estabel ecidas para esse ti po de serviço pela OPS/OMS; a acessi bilidade e o padrão de utilização foram avaliados tomando-se por base inquérito populacional com os adolescentes da área. Os resultados apontaram: bom grau de adequação do programa à sua fi nali dade, necessitando ajustes, especial mente no item recursos humanos; inexi stência de barrei ras de acesso dos usuários ao servi ço; cobertura das ações satisfatória, embora com baixa concentração de atendi mentos por adolescente; padrão de utilização compatível com o perfil de oferta, direcionado principalmenteà assi stência curativa eindividual. Recomendações são feitas no senti do de reverter o model o assi stencial prevalecente, de modo a enfatizar ativi dades preventivas, coletivas e educativas com mai or potencial de efetivi dade para a promoção da saúde da população adol escente. Palavras-chave Saúde do Adol escente; Serviços de Saúde; Avaliação; Cobertura de Serviços de Saúde 
Introdução

Segundo a Organização Mundial de Saúde (OMS), a adolescência é uma etapa evolutiva caracterizada pelo desenvolvimento biopsicossocial, delimitada pela faixa entre 10 e 19 anos que, em geral, se inicia com as mudanças corporais da puberdade e termina com a inserção social, profissional e econômica (Silber et al., 1992).

Nos países em desenvolvimento, há cerca de quatro décadas vem sendo reconhecida a importância demográfica da faixa adolescente, visto que este grupo, além de representar aproximadamente $25 \%$ da população geral, tem seus principais riscos à saúde e causas de morbimortalidade relacionados ao meio ambiente, com destaque para as conseqüências da violência, os acidentes e a contaminação por doenças, resultantes das precárias condições de vida e iniqüidade social a que está submetida a maior parte das populações desses países (Ojeda et al., 1985; Guimarães, 1994).

No Brasil, dados da Fundação Instituto Brasileiro de Geografia e Estatística (IBGE) de 1996 revelaram um total de 34 milhões de adolescentes, representando cerca de $21 \%$ da população. No Município de Belém, 23\% da população encontrava-se na faixa etária de 10 a 19 anos (IBGE, 1997).

Tradicionalmente, três critérios são considerados, do ponto de vista da saúde pública, quando se trata de priorizar a assistência à saúde como demanda social: magnitude, transcendência e vulnerabilidade. Entretanto, conforme ressaltam Ayres \& França J r. (1996), não existe uma perspectiva única para formular programas de atenção à saúde do adolescente. Os adolescentes não constituem um grupo homogêneo, mas sim um conjunto de individualidades, e os critérios técnicos não recobrem a totalidade dos interesses e saúde dos adolescentes. Com estas observações, os autores reconhecem que é justificado o desenvolvimento de ações programáticas voltadas à adolescência, por tratar-se de um grupo social que se encontra em fase de importantes transformações biológicas e mentais, articuladas a um redimensionamento de identidades de papéis sociais. O grupo adolescente se caracteriza também por ser fecundo e receptivo à prevenção, às mudanças, ao estímulo para o autoconhecimento e auxílio no seu processo de individuação, maturação e inserção social, especialmente os adolescentes carentes, cujo acesso à informação é precário e ocasional, além de sistema de ensino não contribuir para o seu processo pleno de socialização (Ayres \& França J r., 1996).
As necessidades de saúde na adolescência extrapolam os aspectos orgânico-biológicos, que têm neste grupo pequena expressão como demanda aos serviços de saúde. Desse modo, a apreensão das características sociais da adolescência pode instrumentalizar intervenções com maior potencial de efetividade (Ayres, 1990). Por outro lado, a abordagem multiprofissional que já foi institucionalizada em muitos programas, freqüentemente reduz-se à soma de diferentes saberes, sendo insuficiente para romper com o enfoque clínico-assistencial. Para Ayres (1990), que participa de uma experiência diferenciada de atenção à saúde do adolescente no Centro de Saúde-Escola Samuel Pessoa, em São Paulo, é necessário modificar os pressupostos científico-ideológicos e os métodos desse processo de trabalho coletivo, com o intuito de adequá-los às necessidades específicas do adolescente.

A organização de programas voltados à saúde do adolescente requer, efetivamente, a consideração das dimensões social e coletiva, abordadas de forma multiprofissional e interdisciplinar, envolvendo os diferentes aspectos que interagem no cotidiano dos adolescentes e no contexto em que estão inseridos, procurando adaptar os conteúdos desses programas às diferentes modalidades de demandas individuais e coletivas.

A maior ou menor efetividade desses programas, por sua vez, está relacionada, entre outros aspectos, ao seu grau de adequação aos objetivos, à acessibilidade e cobertura da população à qual se destina.

No campo da avaliação em saúde, alguns atributos expressam as condições do serviço/ programa e a interação entre este e seu objeto, sem o qual o processo de trabalho não se realiza, nem são obtidos os resultados pretendidos. A cobertura e a acessibilidade relacionam-se com a disponibilidade e distribuição social dos serviços de saúde (Silva \& Formigli, 1994). Este tipo de avaliação pode ser considerado um prérequisito para a análise das demais características dos serviços, uma vez que, para se discutir a qualidade técnico-científica, efetividade e satisfação do usuário, é necessário que as ações de saúde sejam prel iminarmente oferecidas à população (Silva et al., 1995). Tais atributos ganham especial relevância na nossa realidade, em que o acesso universal aos serviços de saúde, indispensável para garantir a eqüidade no atendimento, ainda que garantido constitucionalmente, não se concretiza para a maioria da população.

A acessibilidade tem sido mais estudada em relação à dimensão geográfica, chegando- 
se à elaboração de modelos que orientam o planejamento da distribuição dos serviços (Unglert et al., 1987; Unglert, 1990). Mas há uma tendência a entendê-la de modo mais amplo, nas suas dimensões geográfica, econômica, cultural e funcional ou organizacional. Daí depreende-se que é um atributo dependente de características do serviço - localização geográfica, modo de funcionamento, acolhimento, custos, horários de atendimento, disponibilidade de profissionais, do usuário -, do poder aquisitivo, do local de moradia, da disponibilidade de tempo - e da relação entre ambos, em que podem ser incluídos os aspectos culturais - normas e técnicas do serviço adequadas aos valores, representações, hábitos e costumes da população.

A cobertura reflete a interação entre os serviços e a população, estando relacionada, ao mesmo tempo, com a disponibilidade do serviço, com o seu grau de acessibilidade e sua utilização pela clientela. O padrão de utilização do serviço é também determinado por fatores relacionados ao serviço e ao usuário. Segundo Ayres (1990), os motivos da procura de um serviço de saúde são expressão, por um lado, das representações que o usuário faz das suas necessidades e, por outro, da interpretação dessas necessidades pel os profissionais de saúde. É importante salientar, entretanto, que o primeiro aspecto sofre a influência do segundo, na medida em que as representações dos usuários encontram-se impregnadas pelo modo como a medicina apreende e interpreta os fenômenos da vida.

Em 1993, em Belém - Pará, na Instituição República de Emaús, entidade filantrópica que há 25 anos presta assistência a crianças e adolescentes de baixo nível sócio-econômico mediante diferentes projetos que envolvem atividades profissionais e profissionalizantes, recreativas, culturais e educacionais, foi construído e implantado um serviço multiprofissional de saúde para atendimento dos adolescentes assistidos pela Instituição.

O Serviço de Saúde de Emaús (SSE) prestava atendimento clínico para adolescentes, prénatal para gestantes adolescentes, planejamento familiar, atendimento de farmácia e fitoterapia, pediatria (prioritariamente para filhos de adolescentes), atendimento de pequenos procedimentos, odontologia curativa e preventiva. Esta iniciativa foi possível com os recursos da Fundação MacArthur - Estados Unidos, por meio da premiação de um Projeto para Saúde, cuja equipe foi formada com profissionais e estagiários da Universidade, Secretarias Estadual e Municipal de Saúde e de Educação.
Com o decorrer da implementação do serviço, foi verificada a necessidade de multiplicar as ações para ampliar a cobertura e obter maior impacto na promoção da saúde e prevenção de agravos entre os adolescentes da comunidade. Dessa forma, a partir de 1997, já com o apoio da Fundação Kellogg, vem sendo desenvolvido outro projeto que tem como objetivos a sensibilização, capacitação e multiplicação das ações de saúde integral na infância e adolescência entre os próprios adolescentes, educadores, professores e líderes da comunidade.

O presente estudo pretende avaliar o Serviço de Saúde de Emaús no triênio 1994-96, no que diz respeito à sua cobertura, adequação, acessibilidade e utilização, com vistas a subsidiar processos de mudanças no atendimento que possam produzir transformações na realidade de saúde dos adolescentes da área.

\section{Metodologia}

\section{Objeto da avaliação}

O SSE tinha como principal objetivo, na sua criação, a implantação de um modelo de atendimento integral, multidisciplinar e adequado à clientela, visando: à melhoria das condições de saúde dos adolescentes da comunidade; à ampliação do conhecimento sobre sexualidade, com vistas à adoção de comportamento sexual responsável; à redução dos índices de gravidez precoce e não planejada.

A população-alvo do programa constituíase de cerca de 1.200 adolescentes (entre 10 e 19 anos) inseridos nos diferentes projetos/atividades desenvolvidos pela Instituição República de Emaús.

Este trabalho faz parte de um projeto mais amplo de avaliação do Serviço de Saúde de Emaús. O modelo desenhado tenta se aproximar da totalidade de um serviço diferenciado de atenção a adolescentes, contemplando: a) análise do contexto em que se insere o serviço de saúde; b) perfis da população de adolescentes da área de abrangência do projeto (populaçãoalvo) e da população atendida nos diferentes serviços da unidade; c) avaliação propriamente dita do serviço, por intermédio dos seguintes atributos: cobertura, adequação, acessibilidade, padrão de utilização, qualidade técnicocientífica, efetividade e satisfação do usuário.

No modelo de avaliação concebido, os quatro elementos que são objeto deste estudo integram um conjunto articulado que se constitui como pré-requisito à avaliação dos demais atributos: adequação, acessibilidade, cobertu- 
ra e padrão de utilização. A adequação está relacionada à disponibilidade do serviço, desde que estruturado e funcionando de acordo com as finalidades às quais se propõe. Por sua vez, a acessibilidade diz respeito à existência ou não de barreiras ao uso do serviço pelo usuário. Satisfeitas estas premissas, busca-se saber em que medida o serviço atende à população-alvo, o que implica verificar a cobertura oferecida e, finalmente, conhecer o padrão de utilização dos serviços por parte da clientela.

\section{Conceituação dos atributos}

Considerou-se adequação o grau de suficiência (quantitativa e qualitativa) da capacidade instalada do serviço em relação à base populacional à qual se destina e de compatibilidade dos requisitos específicos de funcionamento com a finalidade a que se propõe. No caso deste estudo, foi utilizada como referência a noção de "condições de eficiência" da Organização Pan-americana de Saúde (OPS) e da OMS, específica para serviços ambulatoriais de atenção de adolescentes, que corresponde a um "conjunto de características que devem reunir os recursos de um serviço para atender, com eficiência, às necessidades de saúde de uma popuIação, entendendo-se como recursos os humanos, físicos, tecnológi cos e de conhecimentos" (OPS/OMS/ Fundación Kellogg, 1996:13).

Acessibilidade foi definida como a possibilidade de o consumidor obter os serviços de que necessita no tempo oportuno e local apropriado, em quantidade suficiente e a um custo razoável (Vuori, 1988); acesso, para o mesmo autor, corresponde à remoção de obstáculos físicos, financei ros e outros para a utilização dos serviços disponíveis (Vuori, 1991). A acessibilidade foi entendida neste estudo nas suas dimensões geográfica, econômica, cultural efuncional ou organizacional (OPS, 1978 apud Unglert et al., 1987).

Foi utilizado o conceito de cobertura efetiva da OMS (1981:29), o qual corresponde à utilização dos serviços pela população, expressando "a proporção de pessoas que necessitam de um serviço e o recebem, efeti vamente, num determinado período".

O padrão de utilização refere-se à freqüência e à forma como os serviços são utilizados pela clientela, buscando-se entender até que ponto as determinações dessa escolha foram orientadas por necessidades de saúde ou estiveram relacionadas ao próprio padrão de oferta do serviço.
Procedimentos para avaliação dos atributos

- A avaliação da adequação, na sua vertente qualitativa, foi realizada pela comparação das características gerais do serviço de Emaús no ano de 1996, com os critérios recomendados pela OPS/ OMS para avaliação de condições de eficiência dos Serviços de Atenção Ambulatorial de Adolescentes, que envolve os itens: programação e administração; normas e procedimentos; educação para a saúde; serviços da comunidade e participação comunitária; recursos humanos; estrutura física; recursos materiais; insumos e serviços de apoio. Cada uma dessas áreas temáticas é integrada por um elenco de subitens que se constituem em requisitos necessários ao adequado funcionamento do serviço. $O$ atendimento de cada um desses subitens recebe pontuações máximas diferenciadas segundo seu grau de relevância: 2, 3, 5, 10, 15 e 1, 1, 2, 5, 7 pontos, respectivamente, para o cumprimento parcial dos requisitos (OPS/OMS/Fundación Kellogg, 1996). O somatório dos pontos obtidos em cada item é expresso em percentuais para comparação com a soma total desejável. A determinação dos pontos para cada um dos subitens no nosso estudo tomou como referência a pontuação da OPS/ OMS, chegando-se a um percentual total de alcance de cada um dos grandes itens. Estes percentuais, por sua vez, foram classificados arbitrariamente em: a) inadequado - abaixo de 50\%; parcial mente adequado - entre 50 e $70 \%$; adequado - acima de $70 \%$.

A adequação quantitativa do serviço foi analisada por meio da produção de serviços, comparando o número médio de atendimentos efetivamente realizados com aqueles esperados entre os anos de 1994-1996, de acordo com a capacidade instalada de recursos humanos da unidade, utilizando-se os parâmetros de programação adotados pelo Programa de Atenção à Saúde Integral do Adolescente - PROSAD (MS, 1993).

- Foram adotados dois procedimentos para calcular a cobertura:

a) utilizando informações levantadas na unidade de saúde ejunto à Instituição Emaús, mediante os seguintes indicadores:

$$
\begin{gathered}
\text { número de adolescentes inscritos } \\
\text { no serviço entre } 1994 \text { e 1996; } \\
\hline \text { população atendida pela Instituição } \\
\text { Emaús no mesmo período }
\end{gathered}
$$

número médio de atendimentos realizados por ano na unidade de saúde entre 1994 e 1996

população atendida pela Instituição Emaús no mesmo período 
b) calculando-se a taxa de utilização dos serviços nos últimos três meses do ano de 1996, colhida diretamente junto aos adolescentes da área.

- A acessibilidade e o padrão de utilização dos serviços foram avaliados com base em um inquérito populacional realizado com uma amostra de adolescentes, utilizando-se questionário aplicado por pessoal treinado.

Foi realizada uma amostragem aleatória estratificada. Calculou-se que seria necessário estudar 401 adolescentes, considerando-se um número total estimado de 1.200 adolescentes, uma proporção esperada de $50 \%$ (aquela que exigiria o maior " $n$ "), um nível de confiança de $95 \%$, e um grau de precisão de $5 \%$. Os adolescentes foram estratificados conforme o projeto institucional no qual estavam inseridos - Arte deViver, Mercado Formal deTrabal ho, Oratório Festivo, Operário Autônomo, Ciranda, Escola e outros. Tentou-se selecionar aleatoriamente para participar da amostra a mesma proporção de adolescentes encontrada no universo para cada um desses estratos. Contudo, por problemas operacionais, não se obteve esta proporcionalidade pretendida.

Para aferição da acessibilidade foi investigada a existência de barrei ras de ordem geográfica (com perguntas como: qual a distância aproximada entre o SSE e a sua casa? Você utiliza algum meio de transporte?), econômica (Quanto você gasta para vir ao serviço de saúde?) e funcional (Quanto tempo você espera normalmente para ser atendido?). Os dados foram analisados pelos seguintes indicadores: percentual de adolescentes que consideram o serviço próximo ou distante da residência; percentual de adolescentes que referem a utilização ou não de meio de transporte; medianas de tempo gasto para deslocar-se de casa ao serviço de saú de e de tempo de espera para atendimento.

O padrão de utilização foi analisado valendo-se de questões sobre conhecimento a respeito dos serviços oferecidos, freqüência da utilização dos serviços nos últimos três meses, tipos de serviço freqüentados e as razões alegadas para a procura do serviço.

Os dados referentes à adequação e à cobertura foram processados manualmente. Para o estudo da acessibilidade e utilização foi criado um banco de dados no editor de dados do programa Statistical Package for the Social Sciences (SPSS) (Norusis, 1986), e processadas as freqüências simples e as tabulações cruzadas.

\section{Resultados}

\section{Ad equação}

Segundo os critérios estabelecidos, o serviço de Emaús alcançou, em média, cerca de 67,3\% de adequação, situando-se, portanto, como parcialmente adequado. A rubrica recursos humanos teve desempen ho considerado inadequado, os itens serviços da comunidade/ participação comunitária, recursos gerais/serviços de apoio e normas/ procedimentos foram parcialmente adequados e as áreas de programação/ administração, educação para a saúde, recursos materiais e estrutura física obtiveram desempenho satisfatório (Tabela 1).

A unidade possuía no período sete profissionais de saúde - dois pediatras, ginecologista, farmacêutica, psicólogo, odontólogo, auxiliar de enfermagem, com capacidade de atendimento de 11.520 atendimentos/ ano, de acordo com a norma de dois atendimentos/hora adotada pelo PROSAD para acompanhamento do crescimento e desenvolvimento (MS, 1993), e considerando a jornada de trabal ho de cada um dos profissionais. Foram realizados 2.780 atendimentos nos três anos, o que representa, em média, cerca de 927 atendimentos/ano, ou seja, $8 \%$ do esperado.

\section{Acessibilidade}

Foram entrevistados por meio de inquérito 456 adolescentes cuja distribuição por sexo e idade encontra-se na Tabela 2. Dos 449 casos válidos quanto à informação sobre sexo, 247 (55\%) eram do sexo feminino e 202 (45\%) do sexo masculi-

Tabela 1

Desempenho preconizado e observado por áreas temáticas. Serviço de Saúde de Emaús, Belém, Pará, 1996.

\begin{tabular}{lcrc}
\hline Item/Desempenho & Preconizado & \multicolumn{2}{c}{ Observado } \\
& $n$ & $n$ & $\%$ \\
\hline 1) Programação e administração & 110 & 81 & 73,6 \\
2) Normas e procedimentos & 160 & 106 & 66,3 \\
3) Educação para saúde & 150 & 112 & 74,7 \\
4) Serviços da comunidade/ & 120 & 70 & 58,3 \\
Participação comunitária & & & \\
5) Recursos humanos & 150 & 62 & 41,3 \\
6) Estrutura física & 100 & 100 & 100,0 \\
7) Recursos materiais & 100 & 77 & 77,0 \\
8) Recursos gerais e serviços de apoio & 110 & 65 & 59,1 \\
Total & 1.000 & 673 & 67,3 \\
\hline
\end{tabular}


Tabela 2

Adolescentes segundo sexo e idade. Emaús, Belém, Pará, 1996.

\begin{tabular}{|c|c|c|c|c|c|c|c|c|}
\hline \multirow[t]{3}{*}{ Idade } & \multicolumn{6}{|c|}{ Sexo } & \multicolumn{2}{|c|}{ Total } \\
\hline & \multicolumn{2}{|c|}{ Feminino } & \multicolumn{2}{|c|}{ Masculino } & \multicolumn{2}{|c|}{ Ignorado } & & \\
\hline & $n$ & $\%$ & $n$ & $\%$ & $\mathrm{n}$ & $\%$ & $\mathrm{n}$ & $\%$ \\
\hline $10-14$ & 121 & 49,4 & 68 & 34,0 & 1 & 14,3 & 190 & 42,0 \\
\hline $15-16$ & 76 & 31,0 & 84 & 42,0 & 5 & 71,4 & 165 & 36,5 \\
\hline $17-19$ & 48 & 19,6 & 48 & 24,0 & 1 & 14,3 & 97 & 21,5 \\
\hline Total & $245^{*}$ & 100,0 & $200 *$ & 100,0 & 7 & 100,0 & 452 & 100,0 \\
\hline
\end{tabular}

* Não foi obtida a informação sobre a idade de duas adolescentes e dois adolescentes.

no. As medianas das idades foram semel hantes entre os sexos: 14,5 anos para as meninas e 14,9 anos para os meninos.

A questão sobre a percepção da distância do Serviço foi feita apenas para 248 adolescentes que informaram conhecer o SSE, tendo sido obtidas 225 respostas e perdida a informação em 23 casos (9,3\%). Dos 225 adolescentes, 86 (38,2\%) consideraram o Serviço próximo da residência e 32 (14,2\%) o consideraram distante; outros 96 entrevistados (42,7\%) quantificaram a distância em tempo, perfazendo uma mediana de 33,2 minutos gastos para deslocar-se da casa até a unidade de saúde; 11 adolescentes $(4,9 \%)$ não souberam responder à questão.

Dos 248 entrevistados que conheciam o Serviço, 226 responderam sobre o meio de deslocamento: 121 adolescentes não usavam qualquer meio de transporte para ir de casa à unidade de saúde; 105 adolescentes (46,5\%) referiram a utilização de meio de transporte para deslocamento até a unidade de saúde, sendo que cem deles usaram transporte coletivo, gastando em média $\mathrm{R} \$ 0,80$.

O tempo de espera para atendimento foi quantificado por 138 adolescentes, ficando a mediana em torno de 22,5 minutos. Esta redução do universo pode ser explicada pelo fato de nem todos os adolescentes conhecedores do serviço o haverem utilizado em algum momento, não tendo, portanto, a experiência de aguardar $\mathrm{o}$ atendimento.

\section{Cobertura}

- Cálculo com base nos registros do serviço

A unidade informou a inscrição de 1.288 adolescentes nos diferentes serviços, entre 1994 e 1996. Para uma clientela estimada de 1.200 , isto representa $107 \%$ de cobertura nos três anos.
Considerando a média de 927 atendimentos anuais realizados pela unidade de saúde, já citada anteriormente, conclui-se que cada adolescente teve, em média, 0,7 atendimento por ano. Caso seja refeito o cálculo da cobertura considerando a concentração de dois atendimentos/ano recomendada pelo M inistério da Saúde, verifica-se que foram alcançados anualmente $38,6 \%$ dos adolescentes.

\section{- Cálculo com base no inquérito populacional}

A taxa de utilização dos serviços nos últimos três meses informada pelos adolescentes entrevistados foi de 31,8\%. Esta taxa de utilização, transposta para a população de adolescentes e estimada para o ano, corresponderia a 1.508 atendimentos/ano, o que propiciaria uma cobertura de $62,8 \%$ e 1,3 atendimentos/ adolescente, valores acima daqueles encontrados valendo-se dos registros do serviço.

Padrão de utilização

Inicialmente, foi inquirido o grau de conhecimento geral dos adolescentes em relação ao serviço: 434 adolescentes responderam à questão, ocorrendo perda de informação em 22 casos $(4,8 \%)$, sendo 16 do sexo feminino $(6,5 \%)$ e 6 do sexo masculino (3\%). Entre os que responderam, $248(57,1 \%)$ relataram conhecer a unidade de saúde de Emaús. Destes, 141 (56,9\%) eram meninas e 103 (41,5\%) eram meninos, ocorrendo perda de informação sobre sexo em quatro respostas positivas e três negativas.

Na Tabela 3 observa-se que o atendimento clínico revelou-se o serviço mais conhecido, seguido de odontologia e ginecologia. Todos os serviços da unidade, à exceção de farmácia e curativos eram mais conhecidos por adolescentes do sexo feminino. 
Tipos de atendimento oferecidos pelo Serviço de Saúde, segundo percepção dos adolescentes da comunidade. Emaús, Belém, Pará, 1996.

\begin{tabular}{|c|c|c|c|c|c|c|}
\hline \multirow[t]{3}{*}{ Tipos de atendimento } & \multicolumn{4}{|c|}{ Sexo } & \multicolumn{2}{|c|}{ Total } \\
\hline & \multicolumn{2}{|c|}{ Feminino } & \multicolumn{2}{|c|}{ Masculino } & \multirow[b]{2}{*}{$\mathrm{n}$} & \multirow[b]{2}{*}{$\% *$} \\
\hline & $n$ & $\% *$ & $\mathrm{n}$ & $\% *$ & & \\
\hline Clínico & 88 & 64,2 & 52 & 53,1 & 140 & 59,6 \\
\hline O dontológico & 69 & 50,4 & 44 & 44,9 & 113 & 48,1 \\
\hline Ginecológico & 76 & 55,5 & 17 & 17,3 & 93 & 39,6 \\
\hline Farmácia & 33 & 24,1 & 29 & 29,6 & 62 & 26,4 \\
\hline Curativos & 20 & 14,6 & 27 & 27,6 & 47 & 20,0 \\
\hline Planejamento familiar & 2 & 1,5 & 1 & 1,0 & 3 & 1,3 \\
\hline Pré-natal & 3 & 2,2 & - & 0,0 & 3 & 1,3 \\
\hline Não sabe especificar & 12 & 8,8 & 15 & 15,3 & 27 & 11,5 \\
\hline Total de adolescentes & $137 * *$ & & $98 *$ & & $235^{*}$ & \\
\hline
\end{tabular}

* Percentuais calculados sobre o número de adolescentes; alguns destes conheciam mais de um serviço.

** N ão foi obtida esta informação de quatro adolescentes do sexo feminino (2,8\% das adolescentes que conheciam o serviço) e cinco do sexo masculino (4,9\% dos adolescentes que conheciam o serviço).

*** Foram excluídos deste total os sete adolescentes com sexo ignorado.

Houve 424 respostas válidas sobre utilização de serviços; em 32 casos (7\%) não foi obtida a informação, sendo 21 (8,5\%) meninas e 11 $(5,4 \%)$ meninos. Ocorreu ainda perda de informação sobre sexo em sete respostas negativas. Observou-se que 135 entrevistados (31,8\%) informaram ter utilizado a unidade de saúde nos últimos três meses, sendo 86 meninas (38,1\%) e 49 meninos (25,7\%).

$\mathrm{Na}$ Tabela 4 verifica-se que o serviço mais freqüentado foi o atendimento clínico, seguido do ginecológico e odontológico. Os serviços de curativos, odontológico e de farmácia foram mais utilizados por adolescentes do sexo masculino.

O principal motivo de procura do serviço relatado por adolescentes de ambos os sexos foi a percepção de algum agravo à saúde (Tabela 5). As diferenças nos padrões entre os sexos correram por conta de maiores percentuais de motivos de doenças, problemas odontológicos e acidentes entre os meninos, enquanto que gravidez, atendimento aos filhos e anticoncepção são referidos apenas pelas meninas.

\section{Discussão}

Os resultados obtidos indicam que a oferta de serviços da unidade de Emaús apresenta, em geral, uma composição tecnológica, organizacional e funcional parcialmente adequada ao atendimento das necessi dades de saúde da população adolescente, podendo-se inferir que os instrumentos de trabal ho estão, de modo geral, apropriados para o seu objeto específico, segundo o modelo de intervenção estabelecido, e as recomendações da OPS/ OMS para este tipo de serviço. Em relação à estrutura física e de recursos materiais, o Serviço de Saúde de Emaús apresenta boas condições de atender à clientela de crianças e adolescentes, tendo sido construído e equipado com este objetivo. Do mesmo modo, a adequação é satisfatória no que diz respeito à existência de atividades de educação para a saúde e de programação/ administração. Entretanto, a metodologia de avaliação utilizada permitiu a identificação de algumas áreas críticas no serviço.

As insuficiências apontadas nos itens recursos gerais/ serviços de apoio e serviços da comunidade/ participação comunitária refletem, respectivamente, a ausência de um laboratório para apoio diagnóstico na unidade e a insuficiente articulação com os serviços e organizações comunitárias, bem como a falta de participação dessas organizações nas atividades do serviço de saúde.

O baixo desempenho no que se refere aos recursos humanos é explicado pela não disponibilidade permanente de alguns tipos de profissionais - como psicólogo e assistente social - para integrar uma equipe multidisciplinar desejável, bem como a ausência de especialistas para interconsultas - psiquiatra, psico-pedagogo, nutricionista, recreador e outras especialidades médicas. A principal dificuldade é a falta de um vínculo formal institucional que le- 
Tabela 4

Serviços freqüentados pelos adolescentes nos últimos três meses. Emaús, Belém, Pará, 1996.

\begin{tabular}{|c|c|c|c|c|c|c|}
\hline \multirow[t]{3}{*}{ Serviços freqüentados } & \multicolumn{4}{|c|}{ Sexo } & \multicolumn{2}{|c|}{ Total } \\
\hline & \multicolumn{2}{|c|}{ Feminino } & \multicolumn{2}{|c|}{ Masculino } & \multirow[b]{2}{*}{$\mathrm{n}$} & \multirow[b]{2}{*}{$\% *$} \\
\hline & $n$ & $\% *$ & $n$ & $\% *$ & & \\
\hline Clínico & 50 & 59,5 & 27 & 57,4 & 77 & 58,8 \\
\hline Ginecológico & 38 & 45,2 & $4 * *$ & 8,5 & 42 & 32,1 \\
\hline O dontológico & 15 & 17,9 & 12 & 25,5 & 27 & 20,6 \\
\hline Pré-natal & 9 & 10,7 & - & 0,0 & 9 & 6,9 \\
\hline Farmácia & 4 & 4,8 & 4 & 8,5 & 8 & 6,1 \\
\hline Curativos & 2 & 2,4 & 6 & 12,8 & 8 & 6,1 \\
\hline Total de adolescentes & \multicolumn{2}{|c|}{$84 * * *$} & \multicolumn{2}{|l|}{$47 * * *$} & \multicolumn{2}{|c|}{$131 * * * *$} \\
\hline
\end{tabular}

* Percentuais calculados sobre o número de adolescentes; alguns destes freqüentaram mais de um serviço.

** Este achado pode ser atribuído a situações em que o diagnóstico da parceira houvesse demandado

a presença do companheiro (pré-natal, anticoncepção, DST) ou ainda a erros de registro de dados.

Estas hipóteses não puderam ser verificadas.

*** Não foi obtida esta informação de dois adolescentes do sexo feminino (2,3\% das adolescentes

que freqüentaram o serviço) e dois do sexo masculino $(4,1 \%$ dos adolescentes que freqüentaram o serviço).

**** Foram excluídos deste total os sete adolescentes com sexo ignorado.

Tabela 5

Motivos da procura ao Serviço referidos pelos adolescentes. Emaús, Belém, Pará, 1996.

\begin{tabular}{|c|c|c|c|c|c|c|}
\hline \multirow[t]{3}{*}{ Motivos da procura } & \multicolumn{4}{|c|}{ Sexo } & \multicolumn{2}{|c|}{ Total } \\
\hline & \multicolumn{2}{|c|}{ Feminino } & \multicolumn{2}{|c|}{ Masculino } & \multirow[b]{2}{*}{$\mathrm{n}$} & \multirow[b]{2}{*}{$\% *$} \\
\hline & $\mathrm{n}$ & $\% *$ & $n$ & $\% *$ & & \\
\hline Doenças & 26 & 34,2 & 31 & 67,4 & 57 & 46,7 \\
\hline Gravidez & 27 & 35,5 & - & 0,0 & 27 & 22,1 \\
\hline Problemas odontológicos & 7 & 9,2 & 9 & 19,6 & 16 & 13,1 \\
\hline Acidentes & 2 & 2,6 & 7 & 15,2 & 9 & 7,4 \\
\hline Atendimento aos filhos & 8 & 10,5 & - & 0,0 & 8 & 6,6 \\
\hline Anticoncepção & 6 & 7,9 & - & 0,0 & 6 & 4,9 \\
\hline Acompanhamento/O rientação & 3 & 3,9 & 1 & 2,2 & 4 & 3,3 \\
\hline Total de adolescentes & $76 * *$ & & $46^{*}$ & & 122 & \\
\hline
\end{tabular}

* Percentuais calculados sobre o número de adolescentes; alguns destes procuraram o serviço por mais de um motivo. ** Não foi obtida esta informação de dez adolescentes do sexo feminino $(11,6 \%$ das adolescentes

que freqüentaram o serviço) e três do sexo masculino $(6,1 \%$ dos adolescentes que freqüentaram o serviço).

*** Foram excluídos deste total os sete adolescentes com sexo ignorado.

gitime o trabal ho desses agentes na comunidade e estabeleça uma rotina de trabalho cotidiana. Isto se explica pelo fato de Emaús ser um serviço não governamental e dependente em relação a recursos financeiros, determinando a inserção de profissionais de forma quase voIuntarista. Aqueles que têm permanecido de forma ininterrupta no serviço pertencem à Universidade ou à Secretaria de Saúde. Porém, dificuldades operacionais, tais como incompatibilidade de carga horária da Universidade e falta de um acordo formal entre a Comunidade de
Emaús e o sistema público de saúde, no período estudado, dificultava o processo de adesão de outros profissionais e impedia que as atividades fossem executadas sem solução de continuidade.

A análise da produtividade dos recursos humanos, que expressa a adequação quantitativa da prestação do serviço, também sofreu os reflexos dessa situação de instabilidade de vínculo e de carga horária dos profissionais. Considerando uma base populacional estimada de 1.200 adolescentes, caso fosse cumprida a nor- 
ma de dois atendimentos/adolescente/ ano, recomendada pelo PROSAD, os trabalhadores da unidade deveriam realizar, pelo menos, 2.400 atendimentos/ano. Este número representa cerca de duas vezes e meia os 927 atendimentos efetivamente realizados, que correspondem a 0,7 atendimento/adolescente, muito abaixo do parâmetro normatizado. Estes resultados encontram-se subestimados pela não inclusão de al gumas atividades coletivas e educativas realizadas pela equipe, como palestras, trabalhos em grupo e extramuros, não captadas pelos instrumentos de registro rotineiros, utilizados como fontes de dados neste estudo, os quais são historicamente voltados para as atividades individuais. Além disso, a dificuldade de disponibilizar carga horária do pessoal de saúde e dos próprios adolescentes para essas ações, que ocorrem, em grande parte das vezes, em finais de semana, faz com que esse tipo de atividades ainda não seja expressivo no serviço de Emaús.

O recurso a um inquérito populacional para avaliar a acessibilidade e a utilização dos serviços da unidade, apesar das dificuldades que acarreta, teve como objetivo conhecer com maior precisão o padrão de utilização e seus determinantes, visando otimizar os recursos disponíveis para atendimento, aspecto especialmente importante num contexto de escassez de recursos para a saúde.

Neste ponto, deve ser mais uma vez ressaltado que se obteve uma diferente proporcionalidade entre a amostra e o universo, com relação aos diferentes grupos constitutivos dos mesmos. Desse modo, embora o número de adolescentes estudados tenha sido mais do que suficiente, deve-se ter cautela na generalização dos resultados do inquérito para a população de adolescentes da área. No caso deste estudo há ainda que considerar o número de informações não obtidas para alguns dos aspectos investigados. Tais perdas são freqüentes em estudos como este, que se baseiam na memória dos entrevistados, e somam-se àquel as referentes a possíveis falhas dos entrevistadores.

A importância da acessibilidade para a utilização dos serviços tem sido demonstrada em vários estudos. Em inquérito realizado no bairro de Pau da Lima, Salvador, Bahia, por exemplo, as facilidades de acesso foram condicionantes, em maior proporção, das escolhas de determinados serviços de saúde por parte da população. De modo semelhante, as barreiras de acesso foram razões atribuídas para a não utilização de certos serviços (Silva et al., 1995).

Os dados deste estudo sugerem que, conforme a percepção da clientela de adolescen- tes, a localização do serviço de saúde e o tempo de espera não foram obstáculos de acesso ao atendimento. A barreira econômica, aparentemente, também se configurou pouco relevante, tendo em vista o baixo preço do transporte coletivo e a gratuidade do serviço. Estes achados indicam que existe a possibilidade concreta de os adolescentes utilizarem o serviço. No entanto, isto não é suficiente para que ocorra a utilização efetiva, que depende de outros fatores aqui não investigados, relacionados ao serviço (como a presença dos profissionais em horários compatíveis) e ao próprio usuário (motivação, percepção do processo saúde/ doença). Todos estes aspectos irão influenciar em que medida a população-alvo será alcançada pelo serviço (cobertura) e como ela o está utilizando.

O cálculo da cobertura mediante as informações originadas no próprio serviço tem a vantagem de ser facilmente obtido, uma vez que as informações encontram-se disponíveis nas estatísticas da unidade. Mas os registros do serviço apresentam várias e freqüentes imperfeições. As informações obtidas diretamente junto à clientela podem retratar com maior precisão o padrão de utilização dos serviços e os fatores que o condicionam, embora sejam de mais difícil e custosa obtenção. Em ambos os resultados, que não foram coincidentes, foi alcançado um significativo percentual da população-alvo, porém com número de atendimentos insuficiente em relação à norma recomendada, o que compromete a efetividade do programa.

A el evada freqüência de desconhecimento dos serviços pelos entrevistados, bem como a maior referência aos serviços curativos, podem estar revelando a pequena familiaridade dos adolescentes com as ações de caráter coletivo, preventivo e educativo, ou ainda a insuficiência de oferta e divulgação desses serviços. Esta informação contradiz a análise da adequação, que havia detectado um bom desempenho no item educação em saúde, demonstrando que a simples disponibilidade dessas atividades é insuficiente para garantir sua utilização e efetividade. O pequeno número de citações do planejamento familiar é um dado preocupante, tendo em vista a alta freqüência de iniciação sexual precoce e algumas de suas conseqüências, como gravidez e doenças sexualmente transmissíveis na adolescência.

O fato de quase um terço dos adolescentes ter utilizado o SSE nos últimos três meses pode estar influenciado pela então recente inauguração da unidade e pela demanda reprimida até aquela data. Todavia, não existem padrões 
semelhantes para comparação. Ayres (1990), estudando demanda de adolescentes no setor de atendimento ao adulto do Centro de SaúdeEscola Samuel B. Pessoa, em São Paulo, entre os anos de 1978 e 1984, observou que, excetuando-se a condição específica da gravidez, o grupo etário situado entre os 10 e 20 anos apresenta, em média, um baixo grau de objetivação de demanda, do ponto de vista do instrumental clássico da medicina, ou seja, a faixa adolescente utiliza pouco o serviço de saúde, porque são poucas as necessi dades interpretadas por esses serviços de saúde para ela. Há que se considerar, entretanto, que aqui se trata de um serviço criado e organizado exclusivamente para esta clientela, donde se espera que um dos seus objetivos seja, exatamente, a interpretação das suas necessidades, ampliando, portanto, a demanda/ captação de clientela.

Este estudo apontou que os adolescentes conheciam e utilizaram, principalmente, atendimentos de caráter predominantemente curativo. Ressalve-se, contudo, a possibilidade de que, no caso da escassa referência ao planejamento familiar, além da real desinformação sobre esse serviço, este esteja sendo considerado como atendimento de ginecologia, assim como o pré-natal, visto que as ações são realizadas pelo mesmo profissional. Reforça esta última hipótese o número elevado de citações da gravidez como motivo de procura ao serviço.

O maior grau de conhecimento e utilização dos serviços pelas meninas denota uma maior preocupação e cuidados com a saúde pela população feminina no grupo adolescente, como já é observado em outras faixas etárias.

A relativa maior importância de atendimentos curativos e acidentes entre os adolescentes do sexo masculino, apesar dos baixos valores obtidos, pode estar relacionada a um maior grau de exposição dos mesmos a estes riscos, pelo tipo de atividade desenvolvida.

O padrão de utilização do Serviço de Saúde de Emaús apresentou-se compatível com o padrão de informação dos adolescentes sobre o serviço e com os motivos por eles alegados para a procura, predominantemente de atenção curativa, refletindo, por sua vez, o perfil de oferta da unidade, que enfatiza o atendimento da demanda espontânea e esteve atrelado à presença mais constante de determinados profissionais na unidade. Estes achados contrapõemse aos preceitos básicos do atendimento à população adolescente que requerem a conscientização quanto à importância da atenção à saúde integral e busca de orientações quanto à manutenção da saúde.

\section{Comentários finais}

Esta etapa do processo de avaliação reúne quatro elementos inter-relacionados e interdependentes, que dizem respeito às características do serviço e à sua interação com a clientela. É importante para saber se estão dadas as précondições para o funcionamento do serviço e se ele está sendo efetivamente utilizado pela população à qual se destina. O desempenho desses atributos vai incidir diretamente sobre o grau de eficiência e de efetividade do programa, sendo seus resultados importantes para orientar mudanças na estratégia de intervenção, ajustando-a às necessidades da população-alvo e finalidades do programa, bem como para otimizar a sua utilização.

Os problemas relativos a recursos humanos encontrados no SSE podem ser equacionados valendo-se do estabelecimento de parcerias com as Secretarias Estadual e Municipal de Saúde e Universidades, além do avanço do processo de municipalização dos serviços, que pode propiciar a adequação quantitativa e qualitativa dos profissionais.

Apesar de o programa de Emaús se propor a priorizar a prevenção e promoção da saúde, a maior procura dos adolescentes entrevistados por ações curativas revelou a persistência do modelo assistencial clínico, com atendimento médico-odontológico da demanda espontânea, em detrimento das ações dirigidas à promoção e prevenção da saúde. Logo, é recomendável a reorientação das atividades, direcionando a oferta para um maior investimento na realização das ações de promoção da saúde, de caráter coletivo, preventivo e educativo, incluindo aquelas externas à unidade, para superar o modelo assistencial ainda predominante. A reversão desse modelo é especial mente relevante para alcançar uma clientela que não costuma expressar em demanda ao serviço as suas necessidades de saúde

Nesse sentido, é fundamental que a equipe de saúde envide esforços para informar os adolescentes sobre os objetivos do programa e disponibilidade dos profissionais, assim como as atividades desenvolvidas no serviço, principalmente aquelas preventivas e de promoção da saúde, com ênfase à saúde reprodutiva, acompanhamento pré-natal e dos filhos dos adolescentes. Desse modo, buscar-se-á reverter o tipo de informação predominante e estimular a procura daquelas atividades dirigidas à melhoria da qualidade de vida e saúde, inclusive por intermédio da expansão das atividades extramuros junto às famílias, integradas com escolas, projetos, trabal ho e comunidade, em geral. 


\section{Agradecimento}

Os autores agradecem a Adriana Carvalho Meira, exgraduanda de Medicina da Universidade Federal da Bahia, pelo trabalho de digitação e processamento dos dados.

\section{Referências}

AYRES, J. R. C. M., 1990. Adolescência e saúde coletiva: Aspectos epistemológicos da abordagem programática. In: Programação em Saúde Hoje (L. B. Schraiber, org.), pp. 139-182, São Paulo: Editora Hucitec.

AYRES, J. R. C. M. \& FRANÇA Jr., I., 1996. Saúde do Adolescente. In: Saúde do Adulto. Programas e Ações na Unidade Básica (L. B. Schraiber, M. I. B. Nemes \& R. B. M. Gonçalves, org.), pp. 66-85, São Paulo: Editora Hucitec.

GUIM ARÃES, M. H. P., 1994. Gravidez na Adolescência: Seus Determinantes e Conseqüências. Dissertação de Mestrado, Salvador: Instituto de Saúde Coletiva, Universidade Federal da Bahia.

IBGE (Fundação Instituto Brasileiro de Geografia e Estatística), 1997. Contagem da População 1996. Sistema de Recuperação de Informações Municipais. CD-ROM. Rio de Janeiro: IBGE.

MS (Ministério da Saúde), 1993. Normas de Atenção Integral à Saúde do Adolescente. Volume I. Diretrizes Gerais para o Atendimento de Adolescentes. Acompanhamento e Desenvolvimento. Brasília: MS.

NORUSIS, M. J., 1986. SPSS/PC + for the IBM PC/XT/ AT. Chicago: SPSS Inc.

OJEDA, E. N. S.; ROBERTS, E.; KARIN, D. \& CUMINSKY, M., 1985. Adolescencia y juventud: Aspectos demográficos y epidemiológicos. In: La Salud del Adolescente y el Joven en las Americas. Publicación Científica 489. Washington, D.C.: Organización Panamericana de la Salud.

OMS (Organización Mundial de la Salud), 1981. Preparación de Indicadores para Vigilar los Progresos Realizados en el Logro de la Salud para Todos en el Año 2000. Genebra: OMS.
OPS (Organización Panamericana de la Salud)/ OMS (Organización Mundial de la Salud)/FUNDACION KELLOGG, 1996. Condiciones de eficiencia. In: Evaluación de Servicios de Atención Ambulatoria deAdolescentes. Estimación deComplejidad. Condiciones de Eficiencia (OPS, OMS \& Fundación Kellogg, eds.), pp. 13-41, Washington, D.C.: OPS/OMS/Fundación Kellogg.

SILBER, T. J.; MUNIST, M. M.; MADDALENO, M. \& OJEDA, E. N. S., 1992. Manual de Medicina dela Adolescencia. Washington, D.C.: Organización Panamericana de la Salud/Organización Mundial de la Salud.

SILVA, L. M. V. \& FORMIGLI, V. L. A., 1994. Avaliação em saúde: Limites e perspectivas. Cadernos de Saúde Pública, 10:80-91.

SILVA, L. M. V.; FORMIGLI, V. L. A.; CERQUEIRA, M. P.; KRUCHEVSKY, L.; TEIXEIRA, M. M. A.; BARBOSA, A. S. M.; CONCEIÇÃO, P. S. A.; KHOURI, M. A. \& NASCIMENTO, C. L.,1995. O processo de distritalização e utilização de serviços de saúde - Avaliação do caso de Pau da Lima, Salvador, Bahia, Brasil. Cadernos de Saúde Pública, 11:72-84.

UNGLERT, C. V. S., 1990. O enfoque da acessibilidade no planejamento da localização e dimensão dos serviços de saúde. Revista de Saúde Pública, 24: 445-462.

UNGLERT, C. V. S.; ROSENBURG, C. P. \&JUNQUEIRA, C. B., 1987. Acesso aos serviços de saúde - Uma abordagem de Geografia em Saúde Pública. Revista de Saúde Pública, 21:439-446.

VUORI, H., 1988. El concepto de calidad. La calidad de los servicios sanitarios. In: El Control de Calidad en los Servicios Sanitarios: Conceptos y Metodología (H. Vuori, ed.), pp. 37-101, Barcelona: Masson.

VUORI, H., 1991. A qualidade da saúde. Divulgação em Saúde para Debate, 3:17-25. 